TITLE:

\title{
Preservation of reachability and observability under sampling with a first-order hold
}

\author{
$\operatorname{AUTHOR}(S)$ :
}

Hagiwara, $T$

\section{CITATION:}

Hagiwara, T. Preservation of reachability and observability under sampling with a firstorder hold. IEEE Transactions on Automatic Control 1995, 40(1): 104-107

\section{ISSUE DATE:}

1995-01

URL:

http://hdl.handle.net/2433/39965

\section{RIGHT:}

(c) 1995 IEEE. Personal use of this material is permitted. However, permission to reprint/republish this material for advertising or promotional purposes or for creating new collective works for resale or redistribution to servers or lists, or to reuse any copyrighted component of this work in other works must be obtained from the IEEE. 
Preservation of Reachability and Observability under Sampling with a First-Order Hold

Tomomichi Hagiwara

\begin{abstract}
This paper gives the necessary and sufficient condition for the reachability of the sampled-data system $S_{1}$ obtained by the discretization of a linear time-invariant continuous-time system with a first-order hold. Equivalence of the reachability and controllability of $S_{1}$ is also shown. Similar results are given also for observability and reconstructibility. It turns out that $S_{1}$ is reachable only if $S_{0}$ is reachable, while $S_{1}$ is observable if and only if $S_{4} l 0$ is observable, where $S_{0}$ is the sampleddata system obtained by the discretization with a zero-order hold of the same sampling period.
\end{abstract}

\title{
I. INTRODUCTION
}

In sampled-data control, hold circuits are used to convert the discrete-time signals from digital compensators into the continuoustime signals to be applied to the continuous-time systems. Hold circuits can be viewed also as filters which attenuate the high-frequency alias spectra generated by sampling continuous-time signals. Typical hold circuits are a zero-order hold and a first-order hold [1], but the former seems to be particularly popular in industrial applications. The primary reason for this is that a zero-order hold can be implemented

Manuscript received October 20, 1993; revised February 20, 1994

The author is with the Department of Electrical Engineering, Kyoto University, Yoshida, Sakyo-ku, Kyoto 606-01, Japan.

IEEE Log Number 9407018 
quite easily by using the function of $\mathrm{D} / \mathrm{A}$ converters while a first-order hold can be implemented only with the aid of some additional analog circuits. Another reason might be that, when viewed as continuoustime filters, the phase lag of a first-order hold is greater than that of a zero-order hold for high-frequency ranges, which seems to be a disadvantage from the point of view of closed-loop stability. However, the latter reason seems to apply mainly in the case when a digital compensator is obtained by a digital redesign method [2] of a continuous-time compensator and closed-loop stability is not necessarily assured theoretically. If we could use a first-order hold in such a way that closed-loop stability can be assured, then it might provide some advantages over a zero-order hold, such as reduction of the intersample ripple of the response.

Based upon the above consideration, the aim of this paper is to provide a basis for the use of a first-order hold in the context of the state-space approach of control system design. For this purpose, we give the necessary and sufficient condition for the reachability of the sampled-data system obtained by the discretization of a linear timeinvariant continuous-time system with a first-order hold. In addition, we show the equivalence of the reachability and controllability of this sampled-data system. Furthermore, we give similar results for observability and reconstructibility. (For the standard definitions of these concepts, see [3].)

\section{Discretization With a First-Order Hold}

We consider the system given by

$$
\frac{d x}{d t}=A_{c} x+B_{c} u, \quad y=C x,
$$

where $A_{c} \in \boldsymbol{R}^{n \times n}, B_{c} \in \boldsymbol{R}^{n \times m}$, and $C \in \boldsymbol{R}^{p \times n}$. Suppose a first-order hold is connected to the input. Then, $u(t)$ is given by

$u(t)=u(k T)+\frac{u(k T)-u(\overline{k-1} T)}{T}(t-k T)$

$$
(k T \leq t<\overline{k+1} T),
$$

where $T$ denotes the sampling period ( $u(k T)$ stands for $u(k T+0)$ ). It should be noted that there is a built-in constraint that the input $u(t)(k T \leq t<\overline{k+1} T)$ depends not only on $u(k T)$ but also on $u(\overline{k-1} T)$, which shows sharp contrast with a zero-order hold. In particular, $u(t)(0 \leq t<T)$ depends on $u(-T)$, which has been determined before $t=0$ and cannot be changed by the compensator at $t=0$.

The resulting sampled-data system can be described by the equation (see [4], [5])

$$
\begin{gathered}
x(\overline{k+1} T)=A x(k T)+B^{+} u(k T)+B^{-} u(\overline{k-1} T), \\
y(k T)=C x(k T)
\end{gathered}
$$

where,

$$
\begin{gathered}
A=\exp \left(A_{c} T\right), \quad B^{+}=\int_{0}^{T}\left(2-\frac{t}{T}\right) \exp \left(A_{c} t\right) B_{c} d t, \\
B^{-}=\int_{0}^{T}\left(\frac{t}{T}-1\right) \exp \left(A_{c} t\right) B_{c} d t .
\end{gathered}
$$

We denote the system (3) by $S_{1}$, which can be rewritten in the form of the ordinary discrete-time state equation as

$$
\begin{gathered}
{\left[\begin{array}{c}
x(\overline{k+1} T) \\
u(k T)
\end{array}\right]=\left[\begin{array}{cc}
A & B^{-} \\
0 & 0
\end{array}\right]\left[\begin{array}{c}
x(k T) \\
u(\overline{k-1} T)
\end{array}\right]+\left[\begin{array}{c}
B^{+} \\
I_{m}
\end{array}\right] u(k T),} \\
y(k T)=\left[\begin{array}{ll}
C & 0
\end{array}\right]\left[\begin{array}{c}
x(k T) \\
u(\overline{k-1} T)
\end{array}\right] .
\end{gathered}
$$

\section{Controllability and Reachability of $S_{1}$}

\section{A. Definitions of Controllability and Reachability and Their Equivalence}

In this subsection, we first give the definitions of the controllability and reachability of $S_{1}$. In view of the discrete-time state equation (5), let us adopt the following definition.

Definition 1: $S_{1}$ is controllable if the pair $\left(A_{1}, B_{1}\right)$ is controllable, where

$$
\left(A_{1}, B_{1}\right):=\left(\left[\begin{array}{cc}
A & B^{-} \\
0 & 0
\end{array}\right],\left[\begin{array}{l}
B^{+} \\
I_{m}
\end{array}\right]\right) .
$$

Now, let us verify that the above formal definition matches our practical control purposes in spite of the built-in constraint of a first-order hold.

If we regard $S_{1}$ simply as an ordinary discrete-time system, then its controllability might be defined as the property that, given any initial condition $x(0)$, there exists a sequence $u(k T)\left(k=0, \cdots,{ }^{\exists} N-1\right)$ such that $x(N T)=0$. However, this is not appropriate, because this property does not reflect real purposes of control. Namely, this definition does not always imply the property that there exists a sequence $u(k T)(k=0, \cdots, N-1, N, \cdots)$ such that $x(t)=0$ $\left({ }^{\forall} t \geq N T\right)$, because of the built-in constraint of a first-order hold as discussed in the previous section. (This can be easily understood if we notice that $u(t)=0(N T \leq t<\overline{N+1} T)$ implies $u(\overline{N-1} T)=$ $u(N T)=0$ from (2).) Therefore, to define controllability, we must require that there exists a sequence $u(k T)\left(k=0, \cdots,{ }^{\exists} N-2\right)$ such that this together with $u(\overline{N-1} T)=0$ implies $x(N T)=0$. Likewise, as discussed in the previous section, $u(t)(0 \leq t<T)$ is constrained by the unprescribable value $u(-T)$. Despite this constraint, $x(N T)$ is required to be made 0 .

From the above consideration, the controllability of $S_{1}$ should be defined as the property that, given any initial conditions $x(0)$ and $u(-T)$, there exists a sequence $u(k T)\left(k=0, \cdots,{ }^{\exists} N-2\right)$ such that this together with $u(\overline{N-1 T})=0$ implies $x(N T)=0$. Obviously, this definition is equivalent to the controllability of the discrete-time system (5). Thus, validity of the above definition is assured.

Similarly, we are led to the following definition (see [6] for details).

Definition 2: $S_{1}$ is reachable if the pair $\left(A_{1}, B_{1}\right)$ is reachable.

Now, in spite of the singularity of $A_{1}$, we can establish the following result (the straightforward proof [6] is omitted here).

Theorem 1: $S_{1}$ is reachable if and only if it is controllable.

\section{B. Condition for Preservation of Reachability}

In this subsection, we study the necessary and sufficient condition for the reachability of $S_{1}$ in terms of $A_{c}, B_{c}$ and $T$. From Definition 2 , it is reachable if and only if

$$
\operatorname{rank}\left[\begin{array}{ccc}
A-i I_{n} & B & B^{+} \\
0 & I_{m}-z I_{m} & I_{m}
\end{array}\right]=n+m \quad\left({ }^{\vee} \approx \in \mathbf{C}\right)
$$

where $B:=B^{+}+B^{-}=\int_{0}^{T} \exp \left(A_{c} t\right) B_{c} d t$. (Note that $(A, B)$ is nothing but the pair of the sampled-data system obtained by the discretization of (1) with a zero-order hold, which we denote by $S_{0}$.) The condition (8) is nothing but the reachability condition for the pair

$$
\left(A_{2}, B_{2}\right):=\left(\left[\begin{array}{cc}
A & B \\
0 & I_{m}
\end{array}\right],\left[\begin{array}{l}
B^{+} \\
I_{m}
\end{array}\right]\right)
$$

This pair can be regarded as the pair obtained by the discretization of the fictitious $T$-dependent continuous-time pair

$$
\left(A_{2 c}, B_{2 c}\right):=\left(\left[\begin{array}{cc}
A_{c} & B_{c} \\
0 & 0
\end{array}\right],\left[\begin{array}{c}
B_{c} \\
I_{m} / T
\end{array}\right]\right)
$$

with a zero-order hold, because $A_{2}=\exp \left(A_{2 c} T\right), B_{2}=$ $\int_{0}^{h} \exp \left(A_{2 c} t\right) B_{2 c} d t$. 
Remark 1: $\left(A_{2 c}, B_{2 c}\right)$ is reachable if and only if a) $\left(A_{c}, B_{c}\right)$ is reachable and b) $A_{c}$ does not have the eigenvalue $-1 / T$.

Since the eigenvalues and left eigenvectors of $A_{2 c}$ are not dependent on $T$, we can apply the necessary and sufficient condition [7] for the reachability of $S_{0}$ to the pair $\left(A_{2 c}, B_{2 c}\right)$. Then, the following theorem is obtained (see Appendix for proof).

Theorem 2: $S_{1}$ is reachable if and only if the following two conditions are satisfied.

a) $S_{0}$ is reachable.

b) $A_{\mathrm{c}}$ does not have the eigenvalue $-1 / T$.

Remark 2: Suppose that we define the stabilizability of $S_{1}$ by the stabilizability of the pair $\left(A_{1}, B_{1}\right)$. Then, the necessary and sufficient condition for the stabilizability of $S_{1}$ is given by the stabilizability of $S_{0}$ (the condition b) can be dropped). Although we can rewrite the reachability/stabilizability of $S_{0}$ as the conditions on $A_{c}, B_{c}$, and $T$ (see [7], and Theorem A.1 in Appendix), we did not do this because the importance of the theorem seems to be much clearer in the present form of the statement.

\section{OBservability and ReConstructibility of $S_{1}$}

As in the preceding section, let us consider how to define the observability and reconstructibility of $S_{1}$, taking account of practical purposes.

If we regard $S_{1}$ simply as an ordinary discrete-time system, then its observability might be defined as the property that its initia state $x(0)$ can be uniquely determined from the input data $u(k T)$ $\left(k=0, \cdots,{ }^{3} N-1\right)$ and the output data $y(k T)(k=0, \cdots, N)$. However, this is not appropriate, because $u(t)(0 \leq t<T)$ cannot be known completely from the knowledge of the above input data, as discussed in Section II, and it is clearly impossible to determine $x(0)$ under this lack of knowledge. Therefore, to define observability, we must assume that $u(t)(0 \leq t<T)$ is also known. This assumption is equivalent to the assumption that $u(-T)$ as well as the above input and output data can be used. Noting that $x(0)$ can be determined uniquely if and only if $\left[x(0)^{T}, u(-T)^{T}\right]^{T}$ can be determined uniquely (if we know $u(-T)$ ), we are led to the following definition.

Definition 3: $S_{1}$ is observable if the pair $\left(C_{1}, A_{1}\right)$ is observable, where

$$
C_{1}:=\left[\begin{array}{cc}
C & 0 \\
0 & I_{m}
\end{array}\right] .
$$

Similarly, we are led to the following definition (see [6] for details).

Definition 4: $S_{1}$ is reconstructible if the pair $\left(\hat{C}_{1}, A_{1}\right)$ is reconstructible, where

$$
\hat{C}_{1}:=\left[\begin{array}{ll}
C & 0
\end{array}\right]
$$

$\left(\hat{C}_{1}, A_{1}\right)$ is reconstructible if and only if $\left(C_{1}, A_{1}\right)$ is reconstructible. Furthermore, in spite of the singularity of $A_{1}$, we can readily show that $\left(C_{1}, A_{1}\right)$ is reconstructible if and only if it is observable. Thus we obtain the following result.

Theorem 3: $S_{1}$ is observable if and only if it is reconstructible.

Now, from Definition 3, $S_{1}$ is observable if and only if

$$
\operatorname{rank}\left[\begin{array}{cc}
A-\approx I_{n} & B^{-} \\
0 & -z I_{m} \\
C & 0 \\
0 & I_{m}
\end{array}\right]=n+m \quad\left({ }^{\forall} \approx \in \mathbf{C}\right) \text {. }
$$

Since $(C, A)$ is the pair of $S_{0}$, we readily obtain the following theorem.

Theorem 4: $S_{1}$ is observable if and only if $S_{0}$ is observable.
Remark 3: Suppose that we define the detectability of $S_{1}$ by the detectability of the pair $\left(C_{1}, A_{1}\right)$. Then, the necessary and sufficient condition for the detectability of $S_{1}$ is given by the detectability of $S_{0}$. The condition for observability/detectability of $S_{0}$ in terms of $C$, $A_{c}$ and $T$ is given by [7] (see also Remark A.1 in Appendix).

\section{CONCLuSION}

In this paper, we studied the use of a first-order hold in the context of the state-space approach of control system design. We first studied how to define the controllability and reachability for the sampled-data system $S_{1}$ obtained by the discretization of a linear time-invariant continuous-time system with a first-order hold, taking account of the built-in constraint of a first order hold. Next, we showed the equivalence of these two concepts for $S_{1}$. Then, we studied the necessary and sufficient condition for the reachability of $S_{1}$ in terms of the parameters of the continuous-time system and the sampling period. We also gave similar results for observability and reconstructibility. In particular, it turned out that $S_{1}$ is reachable only if $S_{0}$ is reachable, while $S_{1}$ is observable if and only if $S_{0}$ is observable, where $S_{0}$ is the sampled-data system for the zeroorder hold case. The compensator design problem under the use of a first-order hold is also studied in [6].

APPENDIX

PROOF OF THEOREM 2

Before proving Theorem 2, we give a more comprehensible statement of the necessary and sufficient condition for the reachability of $S_{0}$ derived in [7].

Let $\lambda\left(A_{c}\right)$ denote the set of the eigenvalues of $A_{c}$. For each $\lambda_{i} \in \lambda\left(A_{c}\right)$, we define

$$
\begin{aligned}
\Lambda\left(\lambda_{i}\right):= & \left\{\lambda \mid \lambda \in \lambda\left(A_{c}\right), \operatorname{Re}(\lambda)=\operatorname{Re}\left(\lambda_{i}\right),\right. \\
& \left.\operatorname{Im}(\lambda)-\operatorname{Im}\left(\lambda_{i}\right)=2 k \pi / T \quad(k=0, \pm 1, \pm 2, \cdots)\right\} .
\end{aligned}
$$

Note that $\lambda_{i} \in \Lambda\left(\lambda_{i}\right)$, and that $\Lambda\left(\lambda_{i}\right)=\Lambda\left(\lambda_{j}\right)$ if $\lambda_{j} \in \Lambda\left(\lambda_{i}\right)$. Our interest is only in the sets $\Lambda\left(\lambda_{i}\right)$ which have at least two elements. Let $\Lambda_{l}(l=1, \cdots, L)$ be such distinct sets, where we assume that $\Lambda_{l}\left(l=1, \cdots, L^{+}(\leq L)\right)$ are the sets corresponding to the eigenvalues with nonnegative real parts $\left(L\right.$ and $L^{+}$might be zero). We denote the elements of $\Lambda_{l}$ by $\lambda_{l k}\left(k=1, \cdots, K_{l}\right)$.

Next, for each $\lambda_{i} \in \lambda\left(A_{c}\right)$, we define

$$
\Gamma\left(\lambda_{i}\right):=\left[\begin{array}{c}
\eta_{i 1}^{T} \\
\vdots \\
\eta_{i \nu_{i}}^{T}
\end{array}\right],
$$

where $\nu_{i}$ denotes the geometric multiplicity of the eigenvalue $\lambda_{i}$ and $\eta_{i k}^{T}\left(k=1, \cdots, \nu_{i}\right)$ the corresponding linearly independent left eigenvectors. That is to say, all the linearly independent left eigenvectors of $A_{t}$ corresponding to the eigenvalue $\lambda_{i}$ form the rows of $\Gamma\left(\lambda_{i}\right)$. We further define

$$
\Gamma\left(\Lambda_{l}\right):=\left[\begin{array}{c}
\Gamma\left(\lambda_{l 1}\right) \\
\vdots \\
\Gamma\left(\lambda_{l K_{l}}\right)
\end{array}\right]
$$

for $l=1, \cdots, L$. That is to say, all the linearly independent left eigenvectors of $A_{c}$ corresponding to the eigenvalues in the set $\Lambda_{l}$ form the rows of $\Gamma\left(\Lambda_{l}\right)$.

Now, we obtain the following theorem, which is merely a restatement of Theorem 2 of [7]. 
Theorem A.1: $S_{0}$ is reachable (respectively, stabilizable) if and only if the following three conditions hold:

a) $\left(A_{c}, B_{c}\right)$ is reachable (respectively, stabilizable).

b) $A_{c}$ does not have the nonzero eigenvalue

$$
j 2 k \pi / T \quad(k= \pm 1, \pm 2, \cdots) .
$$

c) $\Gamma\left(\Lambda_{l}\right) B_{c}$ has full row rank for $l=1, \cdots, L$ (respectively, $\left.l=1, \cdots, L^{+}\right)$

Remark A.I: The conditions for the observability and the detectability of $S_{0}$ are given by the dual of the conditions a) and c) of the above theorem. (This is a restatement of Theorem 3 of [7].)

Remark A.2: Note that $\Gamma\left(\Lambda_{l}\right)$ never contains the left eigenvectors for the zero eigenvalue of $A_{c}$ if the condition b) holds.

Now, we give the proof of Theorem 2.

Proof of Theorem 2: Without loss of generality, we assume that

$$
A_{c}=\left[\begin{array}{cc}
\hat{A}_{c} & 0 \\
0 & Z
\end{array}\right], \quad B_{c}=\left[\begin{array}{l}
\hat{B}_{c 1} \\
\hat{B}_{c 2}
\end{array}\right],
$$

where $\hat{A}_{c}$ is nonsingular and all the eigenvalues of $Z$ are zero. Then, applying the similarity transformation by the matrix

$$
\left[\begin{array}{ccc}
I & 0 & -\hat{A}_{c}^{-1} \hat{B}_{c \cdot 1} \\
0 & I & 0 \\
0 & 0 & I
\end{array}\right]
$$

to the pair (10), we obtain the pair

$$
\left(\left[\begin{array}{ccc}
\hat{A}_{c} & 0 & 0 \\
0 & Z & \hat{B}_{c 2} \\
0 & 0 & 0
\end{array}\right], \quad\left[\begin{array}{c}
\left(I+\hat{A}_{c \cdot}^{-1} / T\right) \hat{B}_{c 1} \\
\hat{B}_{c 2} \\
I / T
\end{array}\right]\right) .
$$

Applying Theorem A.1 to the pair (10) for the conditions a) and b), and to the pair (20) for the condition c), and taking Remarks 1 and A.2 into account, we can obtain the following necessary and sufficient condition for the reachability of $S_{1}$ :

Al) $\left(A_{c}, B_{c}\right)$ is reachable.

A2) $A_{c}$ does not have the eigenvalue $-1 / T$.

B) $A_{c}$ does not have the nonzero eigenvalue

$$
j 2 k \pi / T \quad(k= \pm 1, \pm 2, \cdots) .
$$

C) $\hat{\Gamma}\left(\hat{\Lambda}_{l}\right)\left(I+\hat{A}_{c}^{-1} / T\right) \hat{B}_{c 11}$ has full row rank for $l=1, \cdots, \hat{L}$, where $\hat{\Lambda}_{l}, \hat{\Gamma}\left(\hat{\Lambda}_{l}\right)$ and $\hat{L}$ are defined for $\hat{A}_{\mathrm{c}}$ in a consistent way with the above definitions of $\Lambda_{l}, \Gamma\left(\Lambda_{l}\right)$ and $L$.

Since the rows of $\hat{\Gamma}\left(\hat{\Lambda}_{l}\right)$ are the left eigenvectors of $\hat{A}_{c}$ by definition, under condition $\mathrm{A} 2$ ) the condition $\mathrm{C}$ ) is equivalent to

C') $\hat{\Gamma}\left(\hat{\Lambda}_{l}\right) \hat{B}_{c: 1}$ has full row rank for $l=1, \cdots, \hat{L}$.

In view of the form of (18), the conditions B) and C') are equivalent to the conditions b) and c) of Theorem A.1. Since the condition A1) is the same as the condition a) of Theorem A.1, and since the condition (A2) is the same as the condition b) of Theorem 2, the proof has become complete.

Q.E.D.

\section{ACKNOWLEDGMENT}

The author wishes to express his gratitude to Prof. M. Kimura for motivating the research through discussions.

\section{REFERENCES}

[1] J. T. Tou, Digital \& Sampled-Data Control Systems. New York: McGraw-Hill, 1959.

[2] P. Katz, Digital Control Using Microprocessors. Englewood Cliffs, NJ: Prentice-Hall, 1981.

[3] R. E. Kalman, P. L. Falb, and M. A. Arbib, Topics in Mathematical System Theory. New York: McGraw-Hill, 1969.

[4] T. Mita, Theory of Digital Control. Tokyo: Shoukou Do, 1984 (in Japanese):

[5] T. Hagiwara, T. Yuasa, and M. Araki, "Stability of the limiting zeros of sampled-data systems with zero- and first-order holds," Int. J. Control, vol. 58, no. 6, pp. 1325-1346, 1993.

[6] T. Hagiwara, "Preservation of reachability and observability under sampling with a first-order hold," Technical Report No. 93-10, Automatic Control Engineering Group, Department of Electrical Engineering II, Kyoto University, 1993.

[7] M. Kimura, "Preservation of stabilizability of a continuous timeinvariant linear system after discretization," Int. J. Syst. Sci., vol. 21, no. 1 , pp. $65-91,1990$ 